\title{
EFFECT OF GAMMA IRRADIATION ON SHELF LIFE AND QUALITY OF BROILER MEAT
}

\author{
F.J. Rima, M. Sadakuzzaman, M.A. Hossain, M.S. Ali and M.A. Hashem* \\ Department of Animal Science, Bangladesh Agricultural University \\ Mymensingh-2202, Bangladesh
}

\begin{abstract}
The experiment was conducted on fresh broiler meat treated with ${ }^{60} \mathrm{Co}$ gamma irradiation having dose of $0\left(T_{0}\right), 1\left(T_{1}\right), 2\left(T_{2}\right)$ and 3.5 kGy $\left.\left(T_{3}\right)\right)$ and stored 0,30 and 60 days at $-20^{\circ} \mathrm{C}$ to estimate the effect on proximate, sensory, physicochemical, biochemical and microbial changes in broiler meat. Factorial experiment $(4 \times 3)$ in completely randomized design (CRD) replicated three times per cell was applied to study the variation among treatment combination. Color was changed significantly $(p<0.05)$ with higher irradiation doses. DM, EE, CP was increased, and Ash and $\mathrm{pH}$ was decreased significantly $(p<0.05)$ with higher irradiation doses. Thiobarbituric acid reactive substances (TBARS), Peroxide value (PV), Free fatty acid (FFA), cooking loss was increased significantly $(p<0.05)$ with irradiation level and storage period. Total viable count (TVC), Total coliform count (TCC), Total yeast and mold count $($ TYMC) was significantly $(p<0.05)$ reduced with irradiation level. Considering all traits it can be concluded that irradiation significantly decreased microbial population and maintain nutritional quality of broiler meat. The irradiation dose 2.0 kGy showed most acceptable for microbial population reduction, maintains overall acceptability and for shelf life extension of broiler meat comparison to non-irradiated meat sample.
\end{abstract}

Keywords: Gamma Irradiation, Broiler Meat, Physicochemical and Microbiological Traits

\section{INTRODUCTION}

Poultry meat has a high biological value and it is the most important source of protein. Commercial broiler strains and few indigenous chicken breeds are fulfilling the global demand for broiler meat over the years (Jaturasitha et al., 2008). Broiler meat is also a good source of phosphorus, other minerals, B-complex vitamins and rich in proteins. Radiation processing of fresh meat extends shelf life and protects the consumer against pathogenic bacteria (Al-bachir and Zeinou, 2009). Fresh meat has a

* Corresponding author: hashem_as@bau.edu.bd 
shelf life of 1 day or less at ambient storage temperatures $\left(20-30^{\circ} \mathrm{C}\right)$ (Olaoye, 2010). Irradiation is recognized as an effective, widely applicable food processing technique. Irradiation, as a method of meat preservation, is the most effective technology in the elimination of pathogenic microorganisms without compromising the nutritional properties and sensory quality of food (WHO, 1999). The wholesomeness of irradiated food has been permitted by the World Health Organization (WHO), the Food and Agriculture Organization (FAO), the International Atomic Energy Agency (IAEA), and the US Food and Drug Administration (FDA) (Sohn et al., 2009). In Korea, 20 food items are approved to be treated by irradiation up to $10 \mathrm{kGy}$ for the purpose of inhibition of sprouting, destruction of food borne insects and parasites, delay of physiological ripening, an extension of shelf life, or improvement of food qualities.

Lately Bangladesh is producing 72.60 Lakh MT meat vis-à-vis demand of 72.14 Lakh MT (DLS, 2018) where broiler is contributing more share (around 50\%). It indicates we are in surplus 0.46 Lakh MT meats production. It`s a prime time for Bangladesh to seek exporting opportunities of meat. To overcome the international trade barrier irradiation can be an effective way to increase the shelf life and safety of meats. At present in Bangladesh, no research has yet been carried out on irradiation of broiler meats. Hence, the study was carried out to determine the effect of gamma irradiation on sensory, proximate, biochemical and microbial qualities of broiler meat and to find out the safe level of irradiation dosage on broiler meats to increase the shelf life.

\section{MATERIALS AND METHODS}

\section{Sample collection and processing}

The study was conducted during December, 2017 to November, 2018 in the Department of Animal Science, Bangladesh Agricultural University, Mymensingh. Sample was collected from local market of Mymensingh. Samples were divided into four groups. Each group was exposed to the irradiation dose having of $0\left(\mathrm{~T}_{0}\right), 1\left(\mathrm{~T}_{1}\right)$, $2\left(\mathrm{~T}_{2}\right)$ and $\left.3.5 \mathrm{kGy}\left(\mathrm{T}_{3}\right)\right)$ at Bangladesh Institute of Nuclear Agriculture. Meat sample was irradiated at Cobalt ${ }^{60}$ GC-5000 (BRIT, India) machine, whose central dose rate was $4.29 \mathrm{kGy} / \mathrm{hr}$. Each group was treated with $4.29 \mathrm{kGy}$ for $14 \mathrm{~min}, 28 \mathrm{~min}$ and 35 min $55 \mathrm{sec}$ for giving 1.00, 2.00 and $3.50 \mathrm{kGy}$, respectively.

\section{Proximate Ccomponents}

Proximate components such as Dry Matter (DM), Ether Extract (EE), Crude Protein (CP) and Ash were carried out according to the methods (AOAC, 1995). All determinations were done in triplicate and the mean values were reported.

\section{Physicochemical and bio-chemical assessment}

$\mathrm{pH}$ value of raw meat and cooking loss was measured using $\mathrm{pH}$ meter (Hanna HI99163) from raw meat homogenate. The homogenate was prepared by blending 5 
$\mathrm{g}$ of meat with $10 \mathrm{ml}$ distilled water. FFA value, POV value and TBARS value were determined by (Sharma et al., 2012). All determination was done in triplicate and mean value was reported.

\section{Sensory evaluation}

Sensory evaluation was executed by a trained 6-member panel (color, flavor, tenderness, juiciness and overall acceptability). Prior to sample evaluation, all panelists participated in orientation sessions to familiarize with the scale attributes (color, smell, juiciness, tenderness, overall acceptability) of indigenous chicken meat using an intensity scale. Each sample was evaluated by using a 9-point hedonic scale ( 9 = like extremely, $8=$ like very much, $7=$ like moderately, $6=$ like slightly, $5=$ neither like nor dislike, $4=$ dislike slightly, $3=$ dislike moderately, $2=$ dislike very much and 1 = dislike extremely) (Pena et al. 2016). Sensory evaluation was accomplished at 0 days and repeated at 30 and 60 days, respectively.

\section{Microbial Assessment}

Ten grams of sample were aseptically homogenized after adding $90 \mathrm{~mL}$ of sterile solution in a sterile Stomacher bag for 2 min (BagMixer ${ }^{\circledR}$ 400, Interscience, France). Consequently the diluents were planted onto aerobic plated count agar (Difco Laboratories), incubated at $37^{\circ} \mathrm{c}$ for $45 \mathrm{~h}$. The total number of colonies observed on plate of each sample after incubation was counted and expressed as log of colony forming units per gram ( $\log \mathrm{CFU} / \mathrm{g}$ ).

\section{Statistical Model and Analysis}

The proposed model for the planned experiment was a factorial experiment with two factors A (Treatments) and B (Days of Intervals) is:

$y i j k=\mu+A i+B j+(A B) i j+\varepsilon i j k i=1, \ldots, A ; j=1, \ldots, B ; k=1, \ldots, n$

Where: $y i j k=$ observation $\mathrm{k}$ in level $\mathrm{i}$ of factor $\mathrm{A}$ and level $\mathrm{j}$ of factor $\mathrm{B}$

$\mu=$ the overall mean

$\mathrm{Ai}=$ the effect of level $\mathrm{i}$ of factor $\mathrm{A}$

$\mathrm{Bj}=$ the effect of level $\mathrm{j}$ of factor $\mathrm{B}$

Data were analyzed using SAS Statistical Discovery software, NC, USA. DMRT test was used to determine the significance of differences among treatments means.

\section{RESULTS AND DISCUSSION}

\section{Proximate analysis}

Table 1 shows DM, CP, EE and Ash content at different treatments was 25.68 to $27.22,23.17$ to $24.03,1.94$ to 2.93 and 1.61 to $1.41 \%$, respectively. The range of different days of interval DM, CP, EE and Ash content was 25.51 to 27.51, 23.82 to $23.57,2.23$ to 2.72 and 1.72 to 1.33 , respectively. The result showed that increasing irradiation dose increased DM content significantly $(\mathrm{p}<0.05)$ and also increased with 
shelf life. A similar result was found by Fallah et al. (2010). Crude protein (CP) was significantly $(\mathrm{p}<0.05)$ increased with increasing irradiation doses but significantly $(p<0.05)$ decreased with storage period. Irradiated treated samples had significantly $(\mathrm{p}<0.05)$ higher amounts of EE compared to control group and significantly increased with storage period. Haque et al. (2017) found similar trend in beef irradiation. Ash was significantly $(\mathrm{p}<0.05)$ decreased with higher irradiation doses and also significantly decreased with increased storage period. Similar results were found by Al-Bachir and Zeinou, (2014) where they showed that Ash content of meat was decreased with the increasing level of irradiation dose. There was positive and significant interaction between treatments and days of interval for DM and EE (Table 1).

\section{Physicochemical and bio-chemical properties}

Table 2 shows the range of different treatments of $\mathrm{pH}$ and cooking loss score was 6.15 to 5.99 and, 23.46 to $25.30 \%$. The rage value of different days of interval for $\mathrm{pH}$ and cooking loss was 6.18 to 5.98 and 23.01 to $25.83 \%$, respectively. The $\mathrm{pH}$ value was significantly $(\mathrm{p}<0.05)$ decreased with increasing irradiation doses and also decreased with storage period. Similar result was found by Aftab et al. (2015) in irradiated broiler meat where $\mathrm{pH}$ was slightly decreased as the irradiation doses increased. Cooking loss was significantly $(\mathrm{p}<0.05)$ increased with higher irradiation doses and with storage period. Decrease in cooking loss of irradiated meat samples could be due to the degradation of myofibrillar and structural proteins were found by Sweetie et al. (2015) in irradiated meat samples which are not similar with the present study.

\section{Biochemical properties}

The range of different treatments for FFA, POV and TBARS were 0.36 to $1.22,0.64$ to 0.96 and 0.29 to $0.54 \%$, respectively. The range values of different days of intervals for FFA, POV and TBARS were 0.50 to $0.86,0.73$ to 0.95 and 0.31 to $0.48 \%$ (Table 2). FFA value was significantly $(\mathrm{p}<0.05)$ increased with irradiation level as well as with storage period. Similarly Quattara et al. (2002) showed that gamma irradiation increased lipid oxidation in ground beef samples. In general terms, irradiation accelerates the lipid oxidation process, which is highly significant in foods with a high content of fats and much unsaturated fatty acids, in which numerous free radicals are formed due to this oxidation (O'Bryan et al., 2008). Haque et al. (2017) found same trend in beef irradiation. POV values were increased significantly $(p<0.05)$ with higher irradiation doses as well as storage time. Similarly Chengliang et al. (2017) found that lipid oxidation was promoted due to irradiation. TBARS was significantly $(p<0.05)$ increased with irradiation doses of broiler meat samples and with storage period. Irradiated samples at $3.5 \mathrm{kGy}$ had higher TBARS value than those irradiated at 2 and $1 \mathrm{kGy}$. Gomes and Silva, (2006) suggested that gamma radiation ( 3 and $4 \mathrm{kGy}$ ) increased TBARS values of mechanically deboned broiler meat in first days of frozen storage $\left(-18^{\circ} \mathrm{C}\right)$ which is in agreement with our results. FFA had significantly interaction between treatment and days of interval. 
Table 1: Proximate composition (mean $\pm \mathrm{SE}$ ) of irradiated broiler meat

\begin{tabular}{|c|c|c|c|c|c|c|c|c|c|}
\hline \multirow[t]{2}{*}{ Parameters } & \multirow[t]{2}{*}{ DI } & \multicolumn{4}{|l|}{ Treatments } & \multirow[t]{2}{*}{ Mean \pm SE } & \multicolumn{3}{|c|}{ Level of significance } \\
\hline & & $\mathbf{T}_{0}$ & $\mathbf{T}_{1}$ & $\mathbf{T}_{2}$ & $\mathbf{T}_{3}$ & & Treat & DI & Treat*DI \\
\hline \multirow[t]{4}{*}{ DM (\%) } & 0 & $23.73 \pm 0.024$ & $25.21 \pm 0.176$ & $26.30 \pm 0.017$ & $26.80 \pm 0.023$ & $25.51^{c} \pm 0.06$ & $<.0001$ & $<.0001$ & $<.0001$ \\
\hline & 30 & $25.21 \pm 0.017$ & $26.51 \pm 0.017$ & $26.76 \pm 0.023$ & $27.14 \pm 0.034$ & $26.65^{b} \pm 0.023$ & & & \\
\hline & 60 & $27.09 \pm 0.029$ & $27.58 \pm 0.020$ & $27.65 \pm 0.020$ & $27.74 \pm 0.029$ & $27.51^{\mathrm{a}} \pm 0.025$ & & & \\
\hline & Mean & $25.68^{d} \pm 0.023$ & $26.43^{c} \pm 0.071$ & $26.90^{b} \pm 0.02$ & $27.22^{\mathrm{a}} \pm 0.029$ & & & & \\
\hline \multirow[t]{4}{*}{$\mathrm{CP}(\%)$} & 0 & $23.25 \pm 0.017$ & $23.82 \pm 0.038$ & $24.05 \pm 0.007$ & $24.15 \pm 0.026$ & $23.82^{\mathrm{a}} \pm 0.060$ & $<.0001$ & $<.0001$ & 0.5863 \\
\hline & 30 & $23.15 \pm 0.017$ & $23.69 \pm 0.029$ & $23.82 \pm 0.038$ & $23.98 \pm 0.011$ & $23.66^{b} \pm 0.023$ & & & \\
\hline & 60 & $23.10 \pm 0.012$ & $23.53 \pm 0.029$ & $23.70 \pm 0.014$ & $23.96 \pm 0.029$ & $23.57^{\mathfrak{c}} \pm 0.021$ & & & \\
\hline & Mean & $23.17^{\mathrm{d}} \pm 0.015$ & $23.68^{c} \pm 0.032$ & $23.86^{b} \pm 0.019$ & $24.03^{\mathrm{a}} \pm 0.022$ & & & & \\
\hline \multirow[t]{4}{*}{ EE $(\%)$} & 0 & $1.77 \pm 0.290$ & $2.09 \pm 0.017$ & $2.42 \pm 0.023$ & $2.64 \pm 0.029$ & $2.23^{c} \pm 0.089$ & $<.0001$ & $<.0001$ & $<.0002$ \\
\hline & 30 & $2.09 \pm 0.017$ & $2.21 \pm 0.029$ & $2.67 \pm 0.052$ & $2.92 \pm 0.029$ & $2.44^{b} \pm 0.031$ & & & \\
\hline & 60 & $2.10 \pm 0.030$ & $2.48 \pm 0.023$ & $3.09 \pm 0.017$ & $3.23 \pm 0.023$ & $2.72^{\mathrm{a}} \pm 0.023$ & & & \\
\hline & Mean & $1.94^{\mathrm{d}} \pm 0.11$ & $2.26^{\mathrm{c}} \pm 0.023$ & $2.72^{\mathrm{b}} \pm 0.031$ & $2.93^{\mathrm{a}} \pm 0.027$ & & & & \\
\hline \multirow[t]{4}{*}{$\operatorname{Ash}(\%)$} & 0 & $1.83 \pm 0.026$ & $1.78 \pm 0.017$ & $1.68 \pm 0.023$ & $1.61 \pm 0.017$ & $1.72^{\mathrm{a}} \pm 0.021$ & $<.0001$ & $<.0001$ & 0.2972 \\
\hline & 30 & $1.79 \pm 0.017$ & $1.48 \pm 0.023$ & $1.40 \pm 0.017$ & $1.37 \pm 0.023$ & $1.45^{\mathrm{b}} \pm 0.02$ & & & \\
\hline & 60 & $1.41 \pm 0.017$ & $1.35 \pm 0.023$ & $1.32 \pm 0.023$ & $1.25 \pm 0.017$ & $1.33^{c} \pm 0.021$ & & & \\
\hline & Mean & $1.61^{\mathrm{a}} \pm 0.02$ & $1.54^{\mathrm{b}} \pm 0.021$ & $1.47^{\mathrm{c}} \pm 0.021$ & $1.41^{\mathrm{d}} \pm 0.02$ & & & & \\
\hline
\end{tabular}

Mean in each row having different superscript varies significantly at values $\mathrm{p}<0.05$. Again, mean values having same superscript in each row did not differ significantly at $\mathrm{p}>0.05 \mathrm{~T}_{0}=$ Control group, $\mathrm{T}_{1}=1 \mathrm{kGy}$ irradiated group, $\mathrm{T}_{2}=2 \mathrm{kGy}$ irradiated group $\mathrm{T}_{3}=3.5$ $\mathrm{kGy}$ irradiated group, DI=Days of Intervals, Treat= Treatment, $\mathrm{T} * \mathrm{DI}=$ Interaction of Treatment and Days of Intervals. 
Table 2: Physicochemical and bio-chemical properties (mean $\pm \mathrm{SE}$ ) of irradiated broiler meat

\begin{tabular}{|c|c|c|c|c|c|c|c|c|c|}
\hline \multirow[t]{2}{*}{ Parameters } & \multirow[t]{2}{*}{ DI } & \multicolumn{4}{|l|}{ Treatments } & \multirow[t]{2}{*}{ Mean $\pm \mathbf{S E}$} & \multicolumn{3}{|c|}{ Level of significance } \\
\hline & & $\mathbf{T}_{0}$ & $T_{1}$ & $T_{2}$ & $\mathbf{T}_{3}$ & & Treat. & DI & T*DI \\
\hline \multirow[t]{4}{*}{ Raw pH } & 0 & $6.25 \pm 0.017$ & $6.21 \pm 0.017$ & $6.15 \pm 0.022$ & $6.10 \pm 0.038$ & $6.18^{\mathrm{a}} \pm 0.024$ & $<.0001$ & $<.0001$ & 0.1384 \\
\hline & 30 & $6.21 \pm 0.017$ & $6.09 \pm 0.020$ & $6.02 \pm 0.026$ & $6.00 \pm 0.027$ & $6.07^{b} \pm 0.023$ & & & \\
\hline & 60 & $6.15 \pm 0.017$ & $5.98 \pm 0.023$ & $5.94 \pm 0.015$ & $5.89 \pm 0.015$ & $5.98^{\mathrm{c}} \pm 0.018$ & & & \\
\hline & Mean & $6.19^{\mathrm{a}} \pm 0.017$ & $6.09^{b} \pm 0.020$ & $6.03^{\mathrm{c}} \pm 0.021$ & $5.99^{d} \pm 0.027$ & & & & \\
\hline \multirow[t]{4}{*}{ FFA $(\%)$} & 0 & $0.24 \pm 0.017$ & $0.38 \pm 0.014$ & $0.48 \pm 0.017$ & $0.89 \pm 0.017$ & $0.50^{c} \pm 0.016$ & $<.0001$ & $<.0001$ & $<.0001$ \\
\hline & 30 & $0.38 \pm 0.017$ & $0.49 \pm 0.011$ & $0.68 \pm 0.017$ & $0.49 \pm 0.017$ & $0.69^{b} \pm 0.016$ & & & \\
\hline & 60 & $0.45 \pm 0.014$ & $0.61 \pm 0.015$ & $0.84 \pm 0.011$ & $1.55 \pm 0.017$ & $0.86^{\mathrm{a}} \pm 0.014$ & & & \\
\hline & Mean & $0.36^{d} \pm 0.016$ & $0.49^{c} \pm 0.013$ & $0.67^{b} \pm 0.015$ & $1.22^{\mathrm{a}} \pm 0.017$ & & & & \\
\hline \multirow{4}{*}{$\begin{array}{l}\text { PV } \\
(\text { meq/kg) }\end{array}$} & 0 & $0.55 \pm 0.020$ & $0.72 \pm 0.008$ & $0.78 \pm 0.017$ & $0.87 \pm 0.011$ & $0.73^{c} \pm 0.014$ & $<.0001$ & $<.0001$ & 0.4753 \\
\hline & 30 & $0.72 \pm 0.008$ & $0.82 \pm 0.008$ & $0.93 \pm 0.014$ & $0.97 \pm 0.012$ & $0.83^{b} \pm 0.011$ & & & \\
\hline & 60 & $0.77 \pm 0.020$ & $0.95 \pm 0.020$ & $1.02 \pm 0.017$ & $1.06 \pm 0.046$ & $0.95^{\mathrm{a}} \pm 0.026$ & & & \\
\hline & Mean & $0.64^{d} \pm 0.016$ & $0.83^{\mathrm{c}} \pm 0.012$ & $0.91^{b} \pm 0.016$ & $0.96^{\mathrm{a}} \pm 0.023$ & & & & \\
\hline \multirow{5}{*}{$\begin{array}{l}\text { TBARS } \\
(\mathrm{mg}-\mathrm{MDA} / \mathrm{kg})\end{array}$} & 0 & $0.18 \pm 0.015$ & $0.25 \pm 0.023$ & $0.35 \pm 0.023$ & $0.48 \pm 0.026$ & $0.31^{c} \pm 0.031$ & $<.0001$ & $<.0001$ & 0.0822 \\
\hline & 30 & $0.27 \pm 0.029$ & $0.37 \pm 0.032$ & $0.47 \pm 0.015$ & $0.56 \pm 0.048$ & $0.42^{\mathrm{b}} \pm 0.039$ & & & \\
\hline & 60 & $0.43 \pm 0.016$ & $0.42 \pm 0.02$ & $0.48 \pm 0.020$ & $0.59 \pm 0.008$ & $0.48^{\mathrm{a}} \pm 0.039$ & & & \\
\hline & Mean & $0.29^{d} \pm 0.02$ & $0.35^{\mathrm{c}} \pm 0.025$ & $0.43^{b} \pm 0.028$ & $0.54^{\mathrm{a}} \pm 0.027$ & & & & \\
\hline & 0 & $21.39 \pm 0.243$ & $23.09 \pm 0.793$ & $23.67 \pm 0.103$ & $23.90 \pm 0.074$ & $23.01^{\mathrm{c}} \pm 0.30$ & $<.0001$ & $<.0001$ & $<.0001$ \\
\hline \multirow{3}{*}{$\begin{array}{l}\text { Cooking Loss } \\
(\%)\end{array}$} & 30 & $23.09 \pm 0.079$ & $23.88 \pm 0.092$ & $24.22 \pm 0.120$ & $25.48 \pm 0.233$ & $24.27^{b} \pm 0.13$ & & & \\
\hline & 60 & $25.45 \pm 0.241$ & $23.68 \pm 0.170$ & $25.69 \pm 0.142$ & $26.53 \pm 0.162$ & $25.83^{\mathrm{a}} \pm 0.17$ & & & \\
\hline & Mean & $23.46^{\mathrm{d}} \pm 0.19$ & $24.21^{c} \pm 0.35$ & $24.52^{\mathrm{b}} \pm 0.12$ & $25.30^{\mathrm{a}} \pm 0.16$ & & & & \\
\hline
\end{tabular}

Mean in each row having different superscript varies significantly at values $p<0.05$. Again, mean values having same superscript in each row did not differ significantly at $p>0.05 T_{0}=$ Control group, $T_{1}=1 \mathrm{kGy}$ irradiated group, $T_{2}=, 2 \mathrm{kGy}$ irradiated group $T_{3}=3.5$ $k$ Gy irradiated group, DI=Days of Intervals, Treat= Treatment, $T^{* D I=I n t e r a c t i o n ~ o f ~ T r e a t m e n t ~ a n d ~ D a y s ~ o f ~ I n t e r v a l s . ~}$ 


\section{Sensory evaluation}

Color, flavor, tenderness, juiciness and overall acceptability score at different treatment was 3.72 to $4.05,4.33$ to $4.44,3.44$ to $3.88,3.88$ to 3.88 and 3.88 to 3.33 , respectively (Table 3). The range values of different days of intervals for color, flavor, tenderness, juiciness and overall acceptability score was 4.75 to $3.33,4.33$ to $3.58,4.58$ to $3.00,4.66$ to 3.16 and 4.58 to 3.16 , respectively. Color, tenderness, juiciness and overall acceptability had significantly $(\mathrm{p}<0.05)$ reduced with different storage time. Similar results were found by Souza et al. (2007). Sweetie et al. (2015) found that chicken, lamb and buffalo meat tenderization is increased with irradiation doses. Flavor was decreased with higher irradiation doses and also decreased with storage period. Juiciness was almost same with all irradiation doses and significantly $(p<0.05)$ decreased with storage time. Overall acceptability was almost same with irradiation doses but decreased significantly $(\mathrm{p}<0.05)$ with storage period. Among all treatment groups higher dose $3.5 \mathrm{kGy}$ showed lower value due to higher lipid oxidation of broiler meat. There was no interaction between treatment and days of internal among all parameters.

\section{Microbiological assessment}

Table 4 shows the range of total viable count, coliform count and yeast and mold among different treatments was 4.17 to $1.50,2.17$ to 0.75 and1.73 to 0.85 , respectively. The range value of overall total viable count, coliform count and yeast and mold count among different days of interval was 2.16 to $2.99,1.10$ to 1.51 and 1.02 to 1.42 , respectively. TVC, TCC and TYMC were significantly $(p<0.05)$ decreased with higher irradiation doses but increased with storage period. Similar results were found by Henriques et al. (2013) and Fallah et al. (2008). There was positive and significant interaction between treatments and days of interval for TVC, TCC and TYMC values (Table 4). 
Table 3: Sensory-attributes (mean $\pm \mathrm{SE}$ ) of irradiated broiler meat

\begin{tabular}{|c|c|c|c|c|c|c|c|c|c|}
\hline \multirow[t]{2}{*}{ Parameters } & \multirow[t]{2}{*}{ DI } & \multicolumn{4}{|l|}{ Treatments } & \multirow[t]{2}{*}{ Mean \pm SE } & \multicolumn{3}{|c|}{ Level of significance } \\
\hline & & $\mathbf{T}_{0}$ & $\mathbf{T}_{1}$ & $\mathbf{T}_{2}$ & $\mathbf{T}_{3}$ & & Treat. & DI & $\mathbf{T} * \mathbf{D I}$ \\
\hline \multirow{5}{*}{ Color } & 0 & $4.67 \pm 0.333$ & $4.67 \pm 0.333$ & $4.83 \pm 0.166$ & $4.83 \pm 0.166$ & $4.75^{a} \pm 0.249$ & 0.0908 & $<.0001$ & 0.2293 \\
\hline & 30 & $4.67 \pm 0.333$ & $4.67 \pm 0.333$ & $4.50 \pm 0.288$ & $3.67 \pm 0.333$ & $4.16^{b} \pm 0.321$ & & & \\
\hline & 60 & $2.67 \pm 0.333$ & $3.33 \pm 0.333$ & $3.67 \pm 0.303$ & $3.67 \pm 0.333$ & $3.33^{c} \pm 0.325$ & & & \\
\hline & Mean & $3.72 \pm 0.33$ & $4.22 \pm 0.33$ & $4.33 \pm 0.252$ & $4.05 \pm 0.277$ & & & & \\
\hline & 0 & $4.67 \pm 0.333$ & $4.33 \pm 0.333$ & $4.33 \pm 0.666$ & $4.00 \pm 0.577$ & $4.33 \pm 0.48$ & 0.1836 & 0.1272 & 0.9948 \\
\hline \multirow[t]{4}{*}{ Flavor } & 30 & $4.33 \pm 0.333$ & $4.00 \pm 0.577$ & $3.67 \pm 0.666$ & $3.33 \pm 0.333$ & $3.83 \pm 0.48$ & & & \\
\hline & 60 & $4.00 \pm 0.577$ & $4.00 \pm 0.577$ & $3.33 \pm 0.333$ & $3.00 \pm 0.577$ & $3.58 \pm 0.52$ & & & \\
\hline & Mean & $4.33 \pm 0.41$ & $4.11 \pm 0.49$ & $3.77 \pm 0.56$ & $3.44 \pm 0.49$ & & & & \\
\hline & 0 & $4.67 \pm 0.333$ & $4.67 \pm 0.333$ & $4.67 \pm 0.333$ & $4.33 \pm 0.333$ & $4.58^{a} \pm 0.33$ & 0.4887 & $<.0001$ & 0.6594 \\
\hline \multirow[t]{6}{*}{ Tenderness } & 30 & $4.67 \pm 0.333$ & $3.67 \pm 0.333$ & $3.67 \pm 0.333$ & $3.33 \pm 0.333$ & $3.50^{b} \pm 0.33$ & & & \\
\hline & 60 & $2.33 \pm 0.333$ & $3.33 \pm 0.333$ & $3.00 \pm 0.577$ & $3.33 \pm 0.333$ & $3.00^{b} \pm 0.39$ & & & \\
\hline & Mean & $3.44 \pm 0.33$ & $3.66 \pm 0.33$ & $3.77 \pm 0.41$ & $3.88 \pm 0.33$ & & & & \\
\hline & 0 & $4.33 \pm 0.333$ & $4.67 \pm 0.333$ & $4.67 \pm 0.333$ & $5.00 \pm 0$ & $4.66^{a} \pm 0.25$ & 0.3519 & $<.0001$ & 0.3685 \\
\hline & 30 & $4.67 \pm 0.333$ & $3.33 \pm 0.333$ & $3.67 \pm 0.333$ & $3.33 \pm 0.333$ & $3.50^{b} \pm 0.33$ & & & \\
\hline & 60 & $3.67 \pm 0.333$ & $2.33 \pm 0.333$ & $3.33 \pm 0.333$ & $3.33 \pm 0.333$ & $3.16^{b} \pm 0.33$ & & & \\
\hline \multirow[t]{3}{*}{ Juiciness } & Mean & $3.88 \pm 0.33$ & $3.44 \pm 0.33$ & $3.88 \pm 0.33$ & $3.88 \pm 0.22$ & & & & \\
\hline & 0 & $4.67 \pm 0.333$ & $4.67 \pm 0.333$ & $4.67 \pm 0.333$ & $4.33 \pm 0.333$ & $4.58^{a} \pm 0.33$ & 0.1290 & $<.0001$ & 0.6154 \\
\hline & 30 & $3.33 \pm 0.333$ & $3.67 \pm 0.333$ & $3.67 \pm 0.333$ & $4.67 \pm 0.333$ & $3.50^{b} \pm 0.33$ & & & \\
\hline Overall & 60 & $3.67 \pm 0.333$ & $3.33 \pm 0.333$ & $3.33 \pm 0.333$ & $2.33 \pm 0.333$ & $3.16^{b} \pm 0.33$ & & & \\
\hline Acceptability & Mean & $3.88 \pm 0.33$ & $3.88 \pm 0.33$ & $3.88 \pm 0.33$ & $3.33 \pm 0.33$ & & & & \\
\hline
\end{tabular}

Mean in each row having different superscript varies significantly at values $p<0.05$. Again, mean values having same superscript in each row did not differ significantly at $p>0.05 . T_{0}=$ Control group, $T_{1}=1 \mathrm{kGy}$ irradiated group, $T_{2}=2 \mathrm{kGy}$ irradiated group $T_{3}=$ $3.5 k G y$ irradiated group, DI=Days of Intervals, Treat= Treatment, T*DI=Interaction of Treatment and Days of Intervals. 
Table 4: Microbial population (mean \pm SE) of irradiated broiler meat

\begin{tabular}{|c|c|c|c|c|c|c|c|c|c|}
\hline \multirow[t]{2}{*}{ Parameters } & \multirow[t]{2}{*}{ DI } & \multicolumn{4}{|l|}{ Treatments } & \multirow[t]{2}{*}{ Mean \pm SE } & \multicolumn{3}{|c|}{ Level of significance } \\
\hline & & $\mathbf{T}_{\mathbf{0}}$ & $\mathrm{T}_{1}$ & $\mathbf{T}_{2}$ & $\mathbf{T}_{3}$ & & Treat. & DI & $\mathrm{T} * \mathrm{DI}$ \\
\hline \multirow{5}{*}{$\begin{array}{l}\text { TVC } \\
(\log \text { CFU/g) }\end{array}$} & 0 & $3.69 \pm 0.095$ & $2.35 \pm 0.054$ & $1.65 \pm 0.048$ & $0.97 \pm 0.049$ & $2.16^{c} \pm 0.062$ & $<.0001$ & $<.0001$ & 0.0158 \\
\hline & 30 & $4.18 \pm 0.074$ & $2.80 \pm 0.061$ & $1.68 \pm 0.061$ & $1.59 \pm 0.026$ & $2.61^{b} \pm 0.051$ & & & \\
\hline & 60 & $4.62 \pm 0.120$ & $3.23 \pm 0.037$ & $2.18 \pm 0.061$ & $1.96 \pm 0.052$ & $2.99^{\mathrm{a}} \pm 0.068$ & & & \\
\hline & Mean & $4.17^{\mathrm{a}} \pm 0.089$ & $2.79^{b} \pm 0.050$ & $1.89^{c} \pm 0.056$ & $1.50^{d} \pm 0.042$ & & & & \\
\hline & 0 & $1.92 \pm 0.070$ & $1.11 \pm 0.040$ & $0.80 \pm 0.043$ & $0.60 \pm 0.030$ & $1.10^{c} \pm 0.046$ & $<.0001$ & $<.0001$ & $<.0001$ \\
\hline \multirow{4}{*}{$\begin{array}{l}\text { TCC } \\
(\log \text { CFU/g) }\end{array}$} & 30 & $2.14 \pm 0.029$ & $1.52 \pm 0.037$ & $0.87 \pm 0.029$ & $0.78 \pm 0.008$ & $1.32^{\mathrm{b}} \pm 0.028$ & & & \\
\hline & 60 & $2.48 \pm 0.026$ & $1.74 \pm 0.026$ & $0.97 \pm 0.052$ & $0.88 \pm 0.034$ & $1.51^{\mathrm{a}} \pm 0.035$ & & & \\
\hline & Mean & $2.17^{\mathrm{a}} \pm 0.045$ & $1.45^{\mathrm{b}} \pm 0.034$ & $0.88^{c} \pm 0.041$ & $0.75^{\mathrm{d}} \pm 0.024$ & & & & \\
\hline & 0 & $1.55 \pm 0.048$ & $1.05 \pm 0.040$ & $0.92 \pm 0.023$ & $0.58 \pm 0.020$ & $1.02^{c} \pm 0.033$ & $<.0001$ & $<.0001$ & 0.0103 \\
\hline \multirow{3}{*}{$\begin{array}{l}\text { TYMC } \\
(\log \text { CFU/g) }\end{array}$} & 30 & $1.65 \pm 0.055$ & $1.36 \pm 0.043$ & $1.05 \pm 0.057$ & $0.84 \pm 0.014$ & $1.26^{b} \pm 0.038$ & & & \\
\hline & 60 & $1.82 \pm 0.078$ & $1.49 \pm 0.049$ & $1.25 \pm 0.023$ & $1.15 \pm 0.045$ & $1.42^{\mathrm{a}} \pm 0.049$ & & & \\
\hline & Mean & $1.73^{\mathrm{a}} \pm 0.055$ & $1.30^{b} \pm 0.044$ & $1.07^{\mathfrak{c}} \pm 0.034$ & $0.85^{\mathrm{d}} \pm 0.026$ & & & & \\
\hline
\end{tabular}

Mean in each row having different superscript varies significantly at values $p<0.05$. Again, mean values having same superscript in each row did not differ significantly at $p>0.05 . T_{0}=$ Control group, $T_{1}=1 \mathrm{kGy}$ irradiated group, $T_{2}=2 \mathrm{kGy}$ irradiated group $T_{3}=3.5$ $k$ Gy irradiated group, DI=Day Intervals, Treat $=$ Treatment, $T * D I=$ Interaction of Treatment and Day Intervals 


\section{CONCLUSIONS}

The study revealed that gamma irradiation had significant effect on nutritional quality of broiler meat. Among the treatments, irradiation dose $2.0 \mathrm{kGy}$ showed best results in terms of overall acceptability, microorganisms load and the shelf life extension of broiler meat. It may be concluded that gamma irradiation will enable to deliver the larger amount of high quality broiler meat with extended shelf life.

\section{REFERENCES}

AOAC. (1995). Official method of analysis. $17^{\text {th }}$ edition. Association of Official Analytical Chemists, Washington, D.C. USA.

Aftab, M., Rafaqat, I., Saleem, F., Aftab, B., Abdullah, R., Iqtedar, M., Kaleem, A. Iftikhar, T., and Naz, S. (2015). Enhancement of shelf life and wholesomeness of goat meat by gamma irradiation treatment. International Journal of Bioscience, 7(4): 177-185.

Al-Bachir, M., and Zeinou, R. (2009). Effect of gamma irradiation on microbial load and quality characteristics of minced camel meat. Meat Science, 82: 119-124.

Al-Bachir, M., and Zeinou, R. (2014). Effect of gamma irradiation on the microbial load, chemical and sensory properties of goat meat. Acta Alimentaria, 43(2): 264-272.

Chengliang, L., Lichao, H., Guofeng, J., Sumin, M., Wenmin, W., and Lan, G. (2017). Effects of different irradiation dose treatment on the lipid oxidation, instrumental color and volatiles of fresh pork and their changes during storage. Meat Science, 43(3): 227-232.

DLS. (2018). Department of Livestock Services. Livestock Economy, Dhaka.

Fallah, A.A., Tajik, H., and Farshid, A.A. (2010). Chemical quality, sensory attributes and ultra structural changes of gamma irradiated camel meat. Journal of Muscle Foods, 21: 597-613.

Fallah, A.A., Saei-Dehkordi. S., and Rahnama, M. (2008). Enhancement of microbial quality and inactivation of pathogenic bacteria by gamma irradiation of ready to cook Iranian barbecued chicken. Radiation Physics and Chemistry, 79: 1073-1078.

Gomes, H.A., and Silva, E.N. (2006). Effects of ionizing radiation on mechanically deboned broiler meat during frozen storage. Journal of Radioanal Nuclear Chemistry, 270: 225229.

Haque, M.A., Hashem, M.A., Hossain, M.M., Rima, F.J., and Hossain, A.A. (2017). Effect of gamma irradiation on shelf life and quality of beef. Journal of Meat Science and Technology, 5(2): 20-28.

Henriques, L.S.V., Henry, F.C., Laderia, S.A., and Antonio, I.M.S. (2013). Elimination of coliforms and Salmonella spp. in sheep meat by gamma irradiation treatment. Brazilian Journal of Microbiology, 44(4): 1147-1153.

Jaturasitha, S., Kayan, A., and Michael, W. (2008). Carcass and meat characteristics of male chickens between Thai indigenous compared with improved layer breeds and their crossbred. Arch Tierz Dummerstorf, 51: 283-294. 
O’Bryan, C.A., Crandall, P.G., Ricke, S.C., and Olson, D.G. (2008). Impact of irradiation on the safety and quality of poultry and meat products: A review. Critical reviews in Food Science and Nutrition, 48: 442-457.

Olaoye, O.A., and Onilude, A.A. (2010). Investigation on the potential use of biological agents in the extension of fresh beef in Nigeria. World Journal of Microbiology and Biotechnology, 26: 1445- 1454.

Pena, M.R.1., Nunez-Serrano, J.A., Torrion, J., and Velaquez, F.J. (2016). Are innovations relevant for consumers in the hospitality industry? A hedonic approach for Cuban hotels. Tourism Management, 55: 184-196.

Quattara, B., Giroux, G., Myefsah, R., Smoragiewicz, W., Saucier, L., and Borsa, J. (2002). Microbiological and biochemical characteristics of ground beef as affected by gamma irradiation, food additives and edible coating film. Radiation Physics and Chemistry, 63: 299-304.

Sohn, S.H., Jang, A., Kim, J.K., Song, H.P., Kim, J.H., Lee, M., and Jo, C. (2009). Reduction of irradiation off-odor and lipid oxidation in ground beef by a-tocopherol addition and the use of a charcoal pack. Radiation physics and chemistry, 78: 141-146.

Souza, A.R.M., Arthur, V., and Canniatti-Brazaca, S.G. (2007). Alteracoes provocadas pela irradiação e armazenamento nos teores de ferro heme em carne de frango. Cienc Tecnol Aliment, 27 (2): 303-306.

Sharma, P., Jha, A.B., and Dubey, R.S. (2012). Reactive oxygen species, oxidative damage and Antioxidative defence mechanism is plants under stressful conditions. Journal of Botany, 1-26.

Sweetie, R., Kanatt, Chawla, S.P., and Sharma, A. (2015). Effect of radiation processing on meat tenderization. Radiation Physics and Chemistry, 111: 1-8.

WHO. (1999). Safety and Nutritional Adequacy of Irradiated Food. Geneva: World Health Organization.

Vereschako, G.G., Tshueshova, N.V., Gorokh, G.A., Kozlov, I.G., and Naumov, A.D. (2016). Effect of External Irradiation and Immobilization Stress on the Reproductive System of Male Rats. Radiatsionnaia Biologiia, Radioecologiia, 56: 56-63. 